\title{
Comparison of pulmonary vein reconnection patterns after multielectrode phased radiofrequency- and cryoballoon ablation of atrial fibrillation
}

\author{
Marcus Wieczorek ${ }^{1 *}$, Kiarash Sassani ${ }^{2}$ and Reinhard Hoeltgen ${ }^{2}$
}

\begin{abstract}
Background: Pulmonary vein isolation (PVI) using phased radiofrequency (RF) energy has been shown to be effective in the treatment of atrial fibrillation (AF).

Methods: We characterize and compare pulmonary vein (PV) reconnection at repeat ablation in patients with AF after initially successful PVI using phased RF technology (PVAC) or 2nd generation cryoballoon (CB). Eighty five patients undergoing redo PVI using multielectrode PVAC phased RF catheter and 66 patients after CB PVI were enrolled $9.7 \pm 3.4$ months after the initial ablation procedure.

Results: The percentage of patients with PV reconnection(s) was comparably high between both groups (93\% PVAC and $92 \%$ CB). However, $75 \%$ of all PVs and left common trunks (CTs) isolated with PVAC were reconnected, compared with $52 \%$ reconnections after CB PVI $(p<0.001)$. A mean of $2.79 \pm 1.2 \mathrm{PVs}$ and $C T s /$ patient were reconnected after PVAC PVI compared with $1.97 \pm 0.8$ in CB patients, $p<0.0001$. No patients in the CB group had 4 reconnected PVs, while this pattern of reconnection was observed in $33 \%$ in the PVAC group $(p<0.0001)$. The percentage of patients in the PVAC group with $\geq 3$ reconnected PVs was significantly higher compared with CB patients (56 patients $(66 \%)$ vs. 17 patients $(26 \%), p<0.0001$ ), while the percentage of patients with no PV reconnection was comparably low in PVAC and CB patients (7 and 8\%, respectively). CTs were most frequently reconnected after PVAC PVI (94\%) and left superior PVs after CB ablation (67\%), respectively.

Conclusions: The number of patients with recurrent AF and PV reconnection(s) at redo PVI was comparably high between both groups. However, the extent and distribution of PV reconnections was different in many aspects, indicating more stable atrial lesions after CB PVI compared with PVAC technology.
\end{abstract}

Keywords: Atrial fibrillation, Pulmonary vein isolation, Phased radiofrequency, Cryoballoon, Pulmonary vein reconnection

\footnotetext{
* Correspondence: drmwieczorek@t-online.de

${ }^{1}$ School of Medicine; Department of Cardiology and Electrophysiology,

Witten/Herdecke University, St. Agnes-Hospital Bocholt, Barloer Weg 125,

46397 Bocholt, Germany

Full list of author information is available at the end of the article
}

(c) The Author(s). 2020 Open Access This article is licensed under a Creative Commons Attribution 4.0 International License, which permits use, sharing, adaptation, distribution and reproduction in any medium or format, as long as you give appropriate credit to the original author(s) and the source, provide a link to the Creative Commons licence, and indicate if changes were made. The images or other third party material in this article are included in the article's Creative Commons licence, unless indicated otherwise in a credit line to the material. If material is not included in the article's Creative Commons licence and your intended use is not permitted by statutory regulation or exceeds the permitted use, you will need to obtain permission directly from the copyright holder. To view a copy of this licence, visit http://creativecommons.org/licenses/by/4.0/ The Creative Commons Public Domain Dedication waiver (http://creativecommons.org/publicdomain/zero/1.0/) applies to the data made available in this article, unless otherwise stated in a credit line to the data. 


\section{Background}

There is general consensus about the efficacy of pulmonary vein isolation (PVI) in the treatment of atrial fibrillation (AF) with catheter ablation procedures. Recovery of pulmonary vein (PV) to left atrium (LA) conduction is the typical electrophysiological finding in patients with recurrence of AF after an initial ablation procedure $[1,2]$. Different PV reconnection rates between 19 and $64 \%$ have been reported in the past, depending on the ablation technology and the targeted PVs $[3,4]$. In case of proven reconnection, repeat PVI can result in a significant improvement in long-term ablation success [5].

The purpose of this study was to characterize and compare PV reconnection patterns at repeat electrophysiological study in patients with AF recurrences after initially successful PVI with phased multielectrode radiofrequency technology (PVAC) or 2nd generation cryoballoon $(\mathrm{CB})$. Both have shown to be safe and effective in PVI procedures [6, 7]. While phased RF technology aims to achieve PVI by duty-cycled uni/-bipolar RF energy using a multipolar circumferential catheter, cryoballoon PVI is designed as a true single shot device to create circumferential transmural lesions after transient complete PV occlusion.

\section{Methods}

\section{Patients}

From a group of 965 patients with a first PVI performed in our institution between May 2013 and June 2018 all patients undergoing a second electrophysiology study in our institution for AF recurrences after completing a blanking period of 3 months were evaluated retrospectively for this study to determine and compare the patterns of PV-LA reconnection, if present. PVI was initially performed with PVAC in 620 patients and with $\mathrm{CB}$ in 345. Only patients with regular follow-up visits in our institution until recurrence of AF were recruited for data analysis. Energy sources for first PVI in our institution were multielectrode phased radiofrequency (PVAC or PVAC Gold, Medtronic, CA, USA) in 85 patients and 2nd generation $\mathrm{CB}$ (Arctic Front Advance, Medtronic, MN, USA) in 66 patients. Patients with a need for touch-up ablation during the first procedure $(6 \%$ for PVAC and $5 \%$ for $\mathrm{CB}$ ) were excluded from this study. The ablation technique used for the index procedure was at the operator's discretion.

\section{Index procedure}

\section{Phased radiofrequency $P V I$}

Our general ablation protocol for PVI using the PVAC has been described in detail elsewhere [8-10]. In order to reduce the higher incidence of clinically silent cerebral microembolism by PVAC, [11] a submerged loading of PVAC into the introducer was routinely practiced since November 2013 and care was taken to avoid electrical interactions between PVAC electrodes 1 and 10, as they contribute to the incidence of cerebral microembolism. PVAC and PVAC GOLD are 9-F, over-the-wire, steerable circular mapping and ablation catheters used in combination with a multichannel RF generator (GENius, Medtronic, CA, USA). The system can synchronously apply duty-cycled phased unipolar/bipolar RF energy over 10- (PVAC) or 9 (PVAC GOLD) electrodes, where bipolar refers to adjacent electrodes. After written informed consent, PVAC was inserted into the LA using a long steerable 9Fr sheath (Greatbatch MedicalTM, MN, USA) and directed over a guidewire placed in each targeted PV towards the PV antrum. We recorded bipolar electrograms of PV potentials over all electrodes. Under deep analgosedation, ablation was always started with a 2:1 bipolar/unipolar ablation mode. It was at the operator's decision to convert to a 1:1 mode, if PVI could not be achieved. Several overlapping RF applications, with a maximum duration of $60 \mathrm{~s}$ each, were made while repositioning the PVAC after each application, until all PV potentials were eliminated. PVAC was also used for validation of bidirectional PV conduction block after a waiting period of $30 \mathrm{~min}$.

\section{Cryoballoon PVI}

After written informed consent, $\mathrm{CB}$ ablation was performed under mild conscious sedation, as previously described by our group [10]. A 15- or 20-mm-diameter inner lumen catheter (Achieve $\mathrm{e}^{\mathrm{TM}}$, Medtronic, MN, USA) was placed in each targeted PV to record PV signals before, during and after ablation. Through a steerable sheath placed in LA (FlexCath ${ }^{\mathrm{TM}}$, Medtronic, MN, USA), the $28-\mathrm{mm}$ or $23 \mathrm{~mm}$ CB (Arctic Front Advance ${ }^{\mathrm{Tm}}$, Medtronic, MN, USA) was advanced over the Achieve catheter, inflated, and then directed to each PV ostium. Optimal vessel occlusion was assumed when contrast injection into the PV showed complete contrast retention without any backflow to the atrium. A single freeze protocol with a standard duration of $240 \mathrm{~s}$ at the beginning and later with $180 \mathrm{~s}$ duration was used with a target temperature of $-40{ }^{\circ} \mathrm{C}$ and/or PV isolation within one min. Additional freezes, with different $\mathrm{CB}$ positions were applied if the first freeze was not successful or in the case of nadir temperature greater than $-40{ }^{\circ} \mathrm{C}$. Phrenic nerve stimulation was performed during ablation of the right-sided PVs to minimize the risk of phrenic nerve palsy by a steerable multipolar catheter placed in the superior vena cava (cycle length $1000 \mathrm{~ms}, 15 \mathrm{~mA} / 3.0 \mathrm{~ms}$ pulse width). To distinguish atrial far-field signals from PV potentials, coronary sinus pacing from different sites was performed, if necessary. PVI with verified entrance 
and exit block or absence of any electrical signal for $\geq 30$ min was performed by the achieve catheter [12].

\section{Definition and ablation strategy of left common trunk (CT)} We defined a CT as a bifurcated PV entering the LA contour together at a distance between the virtual border of the LA and the bifurcation of both PVs $>5$ $\mathrm{mm}$.

CT isolation with CB: If an antral occlusion of the CT, validated by contrast injection, could be obtained, a freeze was initiated. If an antral CT occlusion could not be obtained, a sequential ablation approach targeting each PV branch was used.

CT isolation with PVAC: to avoid PV stenosis of the targeted CTs, PVAC was always directed to the antral aspect of the CTs. Multiple PVAC rotations around the CT ostium followed by energy applications were generally performed, until CT isolation was achieved.

\section{Repeat ablation procedure}

All procedures were performed after informed consent and started under conscious sedation. A hexapolar electrode catheter was placed in the coronary sinus (CS) for pacing and bipolar recordings. After transseptal puncture, a long steerable sheath (FlexCath or Greatbatch Medical).

was inserted into the LA to delineate all PVs angiographically. All PVs were then assessed for PV reconnection. PV reconnection was determined at the onset of the procedure using one of the following multipolar circular catheters: $20 \mathrm{~mm}$ inner lumen mapping catheter Achieve (Medtronic, Minneapolis, MN, USA), Lasso (Biosense Webster Inc., Diamond Bar, CA, USA) or Advisor (Abbott, St. Jude Medical, St. Paul, MN, USA), as preferred by the operator. The catheters were placed in each PV with evaluation for PV potentials. Electric isolation of each PVs was confirmed by entrance and exit block, assessed by Achieve, Lasso or Advisor catheter positioned at the PV antrum and/or inside the PV, as necessary.

All PVs/CTs with PV reconnection were targeted for re-isolation with PVAC GOLD, CB or open-irrigated RF-energy point-by point, depending on the operator's preference. Most patients without PV reconnection were offered an individualized antiarrhythmic drug treatment.

\section{Follow-up strategy}

All our patients underwent routine follow-up visits in an outpatient clinic every 3 months after the index ablation procedure or earlier in case of symptoms. In patients with early and/or recurrent symptomatic episodes of AF, antiarrhythmic drug (AAD) therapy was temporarily used and terminated again after completing the blanking period of 3 months or at least 1 week before the first follow-up visit. Amiodarone therapy was terminated immediately after re-ablation. At each follow-up interval, starting 3 months after the initial procedure, a three-day Holter monitor recording was performed without AAD therapy. Patients were asked to contact our hospital in case of ongoing palpitations. We defined freedom from AF as the absence of AF lasting > $30 \mathrm{~s}$.

\section{Statistical analysis}

All continuous variables are reported as mean \pm standard deviation (SD) and were compared by student's t test, Mann Whitney U test or Wilcoxon test. Categorical variables were compared by chi-square or Fisher's exact method, as appropriate. A two tailed $P$ value $<0.05$ was considered to be statistically significant. Statistical analysis was performed using GraphPad Prism software, version 8 .

\section{Results}

\section{Study population}

A total of 151 patients were included in this study with a mean age of $60 \pm 15$ years, male dominance (63\%), moderate LA enlargement $(43 \pm 5 \mathrm{~mm})$ and paroxysmal $\mathrm{AF}$ in $65 \%$. Lone AF was present in $48 \%$ of all patients. Baseline clinical characteristics were similar between both group (Table 1).

\section{Follow-up results}

Average time until recurrence of AF after ablation was $8.5 \pm 3.1$ months for all patients. Recurrence time was significantly shorter for PVAC patients compared with $\mathrm{CB}$ patients $(8.1 \pm 3.4$ vs. $9.1 \pm 2.6, p=0.0066)$. After a mean of $9.7 \pm 3.4$ months, a redo ablation procedure was performed in all 151 patients with a shorter time interval until re-ablation for PVAC- $(9.2 \pm 3.8$ months $)$ compared with $\mathrm{CB}$ patients $(10.4 \pm 2.9$ months $), p=0.0063$. However, mean time delay between redetection of AF and reablation was not different between both groups (1.1 \pm 0.9 months for PVAC and $1.3 \pm 0.9$ for CB). During follow-up, PVAC patients experienced a mean of $2.3 \pm$ 1.2 AF episodes compared with $1.7 \pm 0.8$ episodes for $\mathrm{CB}$ patients $(p=0.0198)$. The rate of sustained AF episodes with a need for electrical cardioversion was comparable (34\% for PVAC, $46 \%$ for CB). While $75 \%$ of the PVAC patients experienced AF recurrences within 4-9 months after the blanking time, only $56 \%$ in the $\mathrm{CB}$ group had recurrences within this period $(p=0.0151)$.

\section{Reconnection patterns at repeat ablation}

Electrophysiological characteristics of both groups at the time of re-ablation are presented in Table 2. In both groups the percentage of patients with PV reconnection(s) was comparably high: $93 \%$ in the PVAC and $92 \%$ in the CB group. 
Table 1 Clinical characteristics of the enrolled patients $(N=151)$

\begin{tabular}{|c|c|c|c|}
\hline & Group $1($ PVAC N = 85) & Group $2($ Cryo $N=66)$ & $p$ value \\
\hline Age (years) & $58 \pm 17[31-72]$ & $63 \pm 12[29-83]$ & ns \\
\hline Male & $56(66)$ & $39(59)$ & ns \\
\hline $\mathrm{CHA}_{2} \mathrm{DS}_{2}$-VASc-score & $2.2[0-5]$ & $2.4[0-5]$ & ns \\
\hline Paroxysmal AF & $58(68)$ & $40(60)$ & ns \\
\hline Left ventricular ejection fraction (\%) & $57 \pm 8$ & $54 \pm 7$ & ns \\
\hline Left atrial diameter (mm) & $43 \pm 4[37-50]$ & $42 \pm 6[36-53]$ & ns \\
\hline No. of antiarrhythmic drugs & $1.4 \pm 1.3[0-3]$ & $1.1 \pm 1.2[0-3]$ & ns \\
\hline Amiodarone therapy & $17(20)$ & $12(18)$ & ns \\
\hline No structural heart disease & $44(52)$ & $28(42)$ & ns \\
\hline Hypertension & $42(49)$ & $38(58)$ & ns \\
\hline Coronary artery disease & $22(26)$ & $15(23)$ & ns \\
\hline Chronic obstructive pulmonary disease & $9(11)$ & $7(11)$ & ns \\
\hline Implantable cardiac devices ${ }^{\mathrm{a}}$ & $6(7)$ & $7(11)$ & ns \\
\hline
\end{tabular}

Data are numbers with mean \pm SD, range [] and \% in (); ns: no significant difference, ${ }^{a}$ pacemaker, implantable cardioverter/defibrillator, event recorder

In the PVAC group, 237 out of 315 initially isolated PVs/CTs (75\%) were reconnected to LA, while 130 out of $250 \mathrm{PVs} / \mathrm{CTs}(52 \%)$ were reconnected in the Cryo group $(p<0.001)$. As a result, a mean of $2.79 \pm 1.2 \mathrm{PVs}$ and CTs/patient were reconnected after PVAC PVI compared with $1.97 \pm 0.8$ in CB patients, $p<0.0001$. $94 \%$ of the CTs -counted as one PV- in the PVAC group were reconnected, in contrast to $60 \%$ in the Cryo group $(p=0.0473)$. Moreover, we found superior- (82 vs $55 \%)$, inferior - (66 vs $49 \%$ ), left-sided - (82 vs $63 \%$ ) and rightsided PVs (69 vs $42 \%$ ) more frequently reconnected in the PVAC group, compared with CB patients.

Table 2 Electrophysiological characteristics at redo procedure

\begin{tabular}{|c|c|c|c|}
\hline & Group $1($ PVAC N = 85) & Group $2(C B N=66)$ & $p$ value \\
\hline Angiographically delineated PVs / CTs & $308 / 17$ & $244 / 11$ & - \\
\hline Targeted PVs / CTs at initial procedure & $302 / 17$ & $242 / 11$ & 1.0000 \\
\hline Isolated PVs / CTs at initial procedure & 298/17 & $240 / 10$ & 1.0000 \\
\hline Pts. with PV reconnections & 79 (93\%) & $61(92 \%)$ & 1.0000 \\
\hline Reconnected PVs and CTs at redo procedure & $237 / 315(75 \%)$ & $130 / 250(52 \%)$ & $<0.0001$ \\
\hline Reconnected left-sided PVs/CTs & 125/152 (82\%) & $75 / 119(63 \%)$ & 0.0005 \\
\hline Reconnected CTs & $16 / 17(94 \%)$ & $6 / 10(60 \%)$ & 0.0473 \\
\hline Reconnected LSPV & $57 / 67(85 \%)$ & $36 / 54(67 \%)$ & 0.0170 \\
\hline Reconnected LIPV & $52 / 68(76 \%)$ & $33 / 55(60 \%)$ & 0.0533 \\
\hline Reconnected right-sided PVs & $112 / 163(69 \%)$ & $55 / 131(42 \%)$ & $<0.0001$ \\
\hline Reconnected RSPV & $63 / 79(80 \%)$ & $29 / 65(45 \%)$ & $<0.0001$ \\
\hline Reconnected RIPV & 49/84 (58\%) & $26 / 66(39 \%)$ & 0.0321 \\
\hline Reconnected superior PVs & $120 / 146(82 \%)$ & $65 / 119(55 \%)$ & $<0.0001$ \\
\hline Reconnected inferior PVs & 101/152 (66\%) & 59/121 (49\%) & 0.0044 \\
\hline PV reconnections per patient \#: & $2.79 \pm 1.2$ & $1.97 \pm 0.8$ & $<0.0001$ \\
\hline Pts. with 4 reconnected PVs & $28(33 \%)$ & $0(0 \%)$ & $<0.0001$ \\
\hline Pts. with 3 reconnected PVs & $28(33 \%)$ & $17(26 \%)$ & 0.2899 \\
\hline Pts. with 2 reconnected PVs & $18(21 \%)$ & $35(53 \%)$ & 0.0001 \\
\hline Pts. with 1 reconnected PV & $5(6 \%)$ & $9(14 \%)$ & 0.2723 \\
\hline Pts. without reconnected PVs & $6(7 \%)$ & $5(8 \%)$ & 1.0000 \\
\hline
\end{tabular}

PTs Patients, CB 2nd Generation cryoballoon, PVs Pulmonary veins, CTs Common trunks, LSPV Left superior pulmonary vein, LIPV Left inferior pulmonary vein, RSPV Right superior pulmonary vein and RIPV Right inferior pulmonary vein. \#: in patients with a reconnected common trunk, CT was considered and counted as one PV 
A detailed analysis of LA-PV reconnections for each PV showed significantly higher reconnection rates in the PVAC group compared with $\mathrm{CB}$ patients for left superior pulmonary veins (LSPVs) ( $85 \%$ vs. $67 \%, p=0.0170$ ), right superior pulmonary veins (RSPV) (79\% vs. $45 \%$, $p<0.0001$ ) and right inferior pulmonary veins (RIPV) ( $58 \%$ vs. $39 \%, p=0.0321)$. In both groups the lowest reconnection rates were observed for the RIPVs (Fig. 1), while the highest rates of reconnection were detected in CTs after PVAC- (94\%) and in LSPVs after CB PVI (67\%).

The distribution of patients with $0,1,2,3$ and 4 reconnected PVs is presented in Table 2 for each group. No patient in the $\mathrm{CB}$ group had 4 reconnected PVs, while this pattern of reconnection was observed in $33 \%$ of the PVAC group $(p<0.0001)$.

As a result, the percentage of patients in the PVAC group with $\geq 3$ reconnected PVs was significantly higher compared with CB patients (56 patients (66\%) vs. 17 patients $(26 \%), p<0.0001)$, while the percentage of patients with no PV reconnection was comparably low in PVAC and $\mathrm{CB}$ patients (7 and $8 \%$, respectively).

If the percentage of patients with PV reconnections at redo procedure is calculated for each vein (Table 3), there were more patients with reconnected right-sided PVs after PVAC PVI compared with CB treated patients (74 vs $44 \%$ for RSPV and 58 vs $39 \%$ for RIPV).

Reconnection patterns for each ablation device at repeat PVI are presented in Table 4a. For both devices we found higher reconnection rates for left- sided PVs compared with the right-sided. Further, PVAC patients had higher reconnection rates in superior- compared with inferior veins. These effects were not influenced by the high reconnection rates of CTs counted as left-sided PV (Table 4b).

\section{Ablation strategy at repeat ablation}

In 79 PVAC patients, PVI re-Isolation was successfully performed with PVAC or PVAC GOLD in 40-, CB in 36- and irrigated RF in 3 patients. $61 \mathrm{CB}$ patients with $\mathrm{PV}$ reconnections were effectively treated with $\mathrm{CB}$ in 42-, PVAC GOLD in 13- and irrigated RF in 6 patients.

\section{Discussion}

\section{Main Findings}

We analyzed LA-PV reconnection patterns in patients with recurrent AF undergoing repeat PVI. Initial PVI had been performed by duty-cycled phased radiofrequency in 85- (PVAC) and 2nd generation $C B$ in 66 patients. After a mean of 9.7 months, a comparably high percentage of patients (93\% PVAC and 92\% CB) had PV reconnection in one or more PVs. However, there were far more reconnected PVs and CTs after PVAC PVI compared with $\mathrm{CB}$ patients and the number of patients with $\geq 3$ reconnected PVs was significantly higher in PVAC patients. CTs and LSPVs were most frequently reconnected after PVI with PVAC and $\mathrm{CB}$, respectively. PV reconnection patterns of both groups were different in many aspects.

\section{PV reconnection patterns after PVAC PVI}

$\mathrm{PV}$ reconnection is the typical finding in patients with recurrent AF after PVI [1]. Different PV reconnection rates in patients with recurrent $\mathrm{AF}$ have been reported in the past for different ablation methods. After PVAC PVI, Rademakers et al. reported a mean of $3.2 \pm 0.7$ reconnected PVs/patient with $46 \%$ of the patients showing reconnections of three PVs. There was no patient without PV reconnection [13]. Similar results after PVAC PVI were reported by Balt et al.: $98 \%$ of all patients had PV reconnection(s) at repeat ablation with
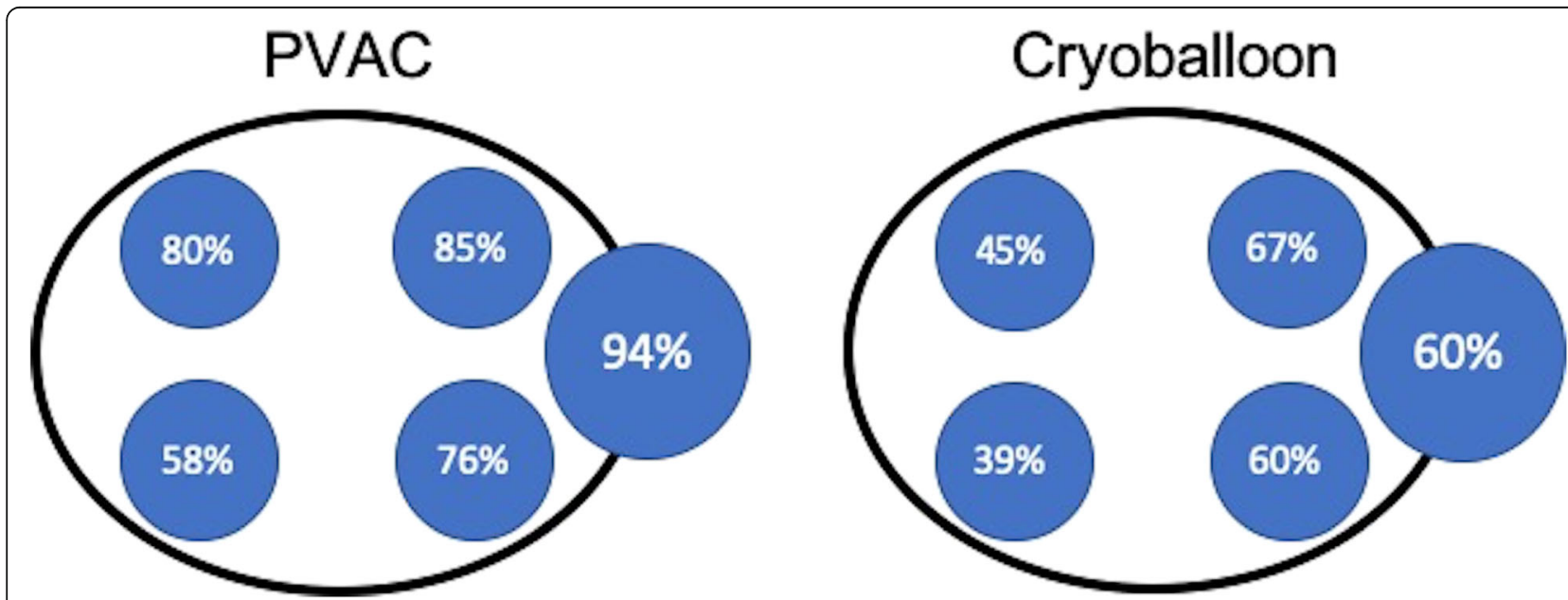

Fig. 1 Schematic left anterior oblique view to LA with percentage of reconnection observed for each pulmonary vein / left common trunk after PVAC (left) and Cryoballoon PVI (right) 
Table 3 Percentage of patients with reconnection at redo procedure calculated for each vein

\begin{tabular}{llll}
\hline & Group 1 (PVAC N=85) & Group 2 (CB N=66) & $p$ value \\
\hline Reconnected CTs & $16(19 \%)$ & $6(9 \%)$ & 0.1077 \\
Reconnected LSPV & $57(67 \%)$ & $36(55 \%)$ & 0.1313 \\
Reconnected LIPV & $52(61 \%)$ & $33(50 \%)$ & 0.1885 \\
Reconnected RSPV & $63(74 \%)$ & $29(44 \%)$ & 0.0002 \\
Reconnected RIPV & $49(58 \%)$ & $26(39 \%)$ & 0.0330 \\
\hline
\end{tabular}

No. of reconnections and percentage () related to each group, CTs Common trunks, LSPV Left superior pulmonary vein, LIPV Left inferior pulmonary vein, RSPV Right superior pulmonary vein and RIPV Right inferior pulmonary vein, CB 2nd Generation cryoballoon

$34 \%$ exhibiting reconnections of three PVs [4]. Similarly, we observed PV reconnections in $93 \%$ of the patients and in $75 \%$ of the previously isolated PVs/CTs after PVAC PVI with a mean of $2.79 \pm 1.2$ reconnected PVs/ CTs per patient. $66 \%$ of our patients had reconnections of three PVs. These results indicate that significant PV/ $\mathrm{CT}$ reconnections must be expected at repeat ablation after PVAC PVI. The highest reconnection rate (94\%) after PVAC PVI was observed for CTs in our study. The same effect was described by Balt et al. with a $83 \%$ reconnection rate for CTs [4]. We further found that right-sided PVs were less frequently reconnected after PVAC PVI compared with left-sided PVs, while superior PVs were more frequently reconnected than inferior veins, both scenarios not influenced by the presence of CTs. As already described by others, the anatomical ridge between LSPV and left atrial appendage (LAA) is a preferred location for PV reconnection, irrespective of the ablation technique $[14,15]$. Reduced contact of the circumferential PVAC at this location in combination with a thicker atrial wall are possible contributors for a higher PV reconnection rate found for the left superior PVs. High reconnection rates found for CTs in our study after PVAC PVI could be explained with the fixed catheter diameter of $25 \mathrm{~mm}$, which can result in inadequate tissue contact in CT diameters beyond $25 \mathrm{~mm}$. To avoid any RF delivery inside the CT, PVAC must be directed antrally to create multiple overlapping positions until CT isolation is achieved.

\section{$\mathrm{PV}$ reconnection patterns after $\mathrm{CB} P \mathrm{PVI}$}

In our study PV reconnections after CB PVI were most frequently observed in LSPVs. This finding is consistent with data presented by Shah et al. and Glowniak et al. $[12,16]$, while Aryana et al. found CTs most frequently reconnected after CB PVI [3]. The anatomical ridge between LSPV and LAA, a common reconnection site with increased wall thickness, reduces the creation of a complete transmural lesion and thus may result in frequent reconnections of the left superior PV. Further, using conventional point-by-point radiofrequency ablation makes is sometimes challenging to obtain stable catheter positions with adequate tissue contact in this area. For cryoballoon ablation an adequate positioning of the balloon in the ridge area resulting in sufficient temperature fall can be difficult to achieve, as well.

$60 \%$ reconnected CTs were observed in our series of patients.

This finding shows the reduced ability of a balloon with a maximum diameter of $28 \mathrm{~mm}$ to routinely achieve a complete occlusion of CTs in all patients. A balloon position more distally inside a CT for sequential isolation its superior and inferior branches, harbors the risk of PV stenosis and may result in smaller antral lesions. Thus, in some of our patients, the proximal portion of the common ostium could have regained electrical connection. Of note, and in contrast to some previous reports of persistent PVI, $[17,18]$ the right inferior PV had the highest isolation rates in our series, yet Westra et al.

Table 4 Comparison of PVAC and CB reconnection patterns

\begin{tabular}{lll}
\hline a) & Reconnected left-sided vs right-sided PVs/CTs & Reconnected superior vs inferior PVs/CTs \\
\hline PVAC & $125 / 152(82 \%)$ vs 112/163 (69\%) & $136 / 163(83 \%)$ vs 101/152 (66\%) \\
p value & $\mathbf{0 . 0 0 6 1}$ & $\mathbf{0 . 0 0 0 6}$ \\
CB & $75 / 119(63 \%)$ vs 55/131 (42\%) & $71 / 129(55 \%)$ vs 59/121 (49\%) \\
p value & $\mathbf{0 . 0 0 1 0}$ & 0.3754 \\
b) & Reconnected left-sided vs right-sided PVs & Reconnected superior vs inferior PVs \\
PVAC & $109 / 135(81 \%)$ vs 112/163 (69\%) & $120 / 146(82 \%)$ vs 101/152 (66\%) \\
P value & $\mathbf{0 . 0 2 3 5}$ & $\mathbf{0 . 0 0 2 3}$ \\
CB & $69 / 109(63 \%)$ vs 55/131 (42\%) & $65 / 119(55 \%)$ vs 59/121 (49\%) \\
P value & $\mathbf{0 . 0 0 1 2}$ & 0.3696 \\
\hline
\end{tabular}


reported $100 \%$ of durable lesions in right inferior PVs in a group of 40 patients after an index CB PVI [19]. A possible explanation could be the routine use of the "hockey stick" and "pull-down" techniques during CB ablation, which was also used in our series of patients, particularly helpful in isolating inferior pulmonary veins [20].

\section{Comparison of PV reconnection patterns (PVAC vs CB)}

While the number of patients with PV reconnections was not different between both groups, more PVs and PVs per patient were found reconnected after PVAC PVI compared with $\mathrm{CB}$ patients. More PVAC patients had more than 2 PVs reconnected at the time of redo PVI. Except for the LIPV ( $p=0.0053)$, we found all other PVs less frequently reconnected after CB PVI. These data suggest, that phased RF energy for PVI creates less stable lesions compared with $\mathrm{CB}$ ablation. The reported higher $\mathrm{PV}$ reconnection rates after conventional radiofrequency PVI compared with $\mathrm{CB}$ ablation, observed by other authors $[3,21]$ can be explained by the mechanism of cryoablation itself: in case of complete PV occlusion, a very uniform lesion surrounding the PV ostium is generally created. As most energy sources for PVI have shown to create transmural lesions, the quality of the lesion is mainly dependent on the application method.

This is the first study to compare the effects of phased radiofrequency, using a circular multipolar catheter (PVAC), on PV reconnection at redo ablation with 2nd generation $\mathrm{CB}$. Reconnection patterns at redo PVI after PVAC ablation were very similar with those reported after point-by point ablation and differ from $\mathrm{CB}$ reconnection patterns in many aspects. Comparable with reported reconnection rates of $67 \%$ after conventional point-by point PVI, [16] we found 75\% PVs reconnected, with multiple PV reconnections per patient and $66 \%$ of the patients (61\%, reported by Glowniak et al. [16]) showing $\geq 3$ reconnected PVs. PVAC lesions around the PV ostium are known to be less homogeneous compared with $\mathrm{CB}$ lesions created with 2nd generation $\mathrm{CB}$, which contributes to the observed differences in PV reconnection patterns. Our data further suggest that $C B$ induced PVI is more persistent over time compared with PVAC technology, although clinical data correlating PV reconnection patterns with freedom from atrial fibrillation are somewhat conflicting.

In a meta-analysis Nery et al. could show that after catheter ablation of AF persistent PVI was associated with a reduced risk of AF recurrence. But the effect was only moderate, and PV reconnections were commonly observed, affecting $58 \%$ of AF-free patients. Other studies of patients with paroxysmal AF even showed a weaker association [22]. Comparable results with 2nd generation $\mathrm{CB}$ were published by Miyazaki et al. They found that the incidence and patterns of PV reconnections after 2nd generation CB ablation were comparable between patients with and those without clinical AF recurrences [23]. It is possible that persistent PVI is not mandatory for all patients to avoid AF recurrence. In a randomized study Dixit et al. compared PVI of only arrhythmogenic PVs with empiric isolation of all PVs.

Study end point was freedom or $>90 \%$ reduction in AF burden while taking previously ineffective antiarrhythmic medication. They found that $75 \%$ of patients randomized to empirical PVI versus $71 \%$ in the arrhythmogenic PV group $(p=0.70)$ had met the endpoint [24]. In contrast, a study of Verma et al. could show that in almost all patients with AF recurrence PV reconnection had occurred. Furthermore, in patients who failed PVI there were more PVs with reconnection(s) compared with patients who maintained in sinus rhythm on antiarrhythmic medication [2]. Irrespective of the uncertainties in the mechanisms of AF, PVI is now a widely accepted endpoint for catheter ablation of AF. However, it remains an anatomic and empirical strategy, rather than a patient-tailored, individual intervention.

\section{Study limitations}

Our study is a retrospective, non-randomized and single-center analysis of AF patients undergoing a second procedure following an index PVI with PVAC or CB. As such, unadjusted confounders may have been present and influenced our results. As this study protocol was non-randomized, we cannot exclude factors which may have confounded either treatment groups. Patients with asymptomatic AF recurrence during follow-up may have been undetected and thus not recruited for analysis. As this would have affected both groups in a comparable way, it is unlikely that this effect could have been a significant source of bias between the two groups. There was no control group of patients without AF recurrences after PVI. Therefore, the pathophysiological impact of our findings with the view of AF recurrences resulting from $\mathrm{PV}$ reconnection alone remains unclear. As there was no adenosine challenge during PV mapping, concealed reconnection might remain undetected in some cases which could have affected the presented PV reconnection patterns in both groups. Further, the reported PV reconnection rates at redo PVI in our study may have been overestimated, as there exist some data about the inaccuracy of PVAC and Achieve mapping catheter to falsely classify some reconnected PVs as isolated $[25,26]$.

\section{Conclusions}

At redo ablation most patients with recurrence of $\mathrm{AF}$ after PAVC and CB PVI had PV reconnection. However, the extent and distribution of reconnected PVs were 
significantly different in many aspects. The use of $\mathrm{CB}$ was associated with greater durability of PV isolation and less frequent PV reconnections/patient compared to PVAC ablation. Whether these differences in PV reconnections affect the rate of AF recurrences has to be determined by appropriate prospective studies.

\section{Abbreviations}

PVI: Pulmonary vein isolation; RF: Radiofrequency; AF: Atrial fibrillation; PV(s): Pulmonary vein(s); PVAC: Phased radiofrequency catheter; CB: 2nd generation cryoballoon; CT(s): Common trunk(s); LA: Left atrium; LAA: Left atrial appendage; LSPV(s): Left superior pulmonary vein(s); LIPV(s): Left inferior pulmonary vein(s); RSPV(s): Right superior pulmonary vein(s); RIPV(s): Right inferior pulmonary vein(s)

\section{Acknowledgements}

None.

\section{Authors' contributions}

MW und RH performed equally invasive studies. KS analyzed and interpreted the patient data for PVAC and MW the data for cryoballoon. MW was the major contributor in writing the manuscript. All authors read and approved the final manuscript.

\section{Funding}

No funding.

\section{Availability of data and materials}

The datasets during and/or analyzed during the current study available from the corresponding author on reasonable request.

\section{Ethics approval and consent to participate}

This study was approved by the local ethics committee of University Witten/ Herdecke, Germany. Written informed consent was obtained from all patients in this study.

\section{Consent for publication}

Not applicable.

\section{Competing interests}

The authors declare that they have no competing interests.

\section{Author details}

${ }^{1}$ School of Medicine; Department of Cardiology and Electrophysiology, Witten/Herdecke University, St. Agnes-Hospital Bocholt, Barloer Weg 125, 46397 Bocholt, Germany. ${ }^{2}$ St. Agnes-Hospital Bocholt, Barloer Weg 125, 46397 Bocholt, Germany.

\section{Received: 28 January 2020 Accepted: 1 April 2020}

Published online: 23 April 2020

\section{References}

1. Ouyang F, Antz M, Ernst S, Hachiya H, Mavrakis H, Deger FT, et al. Recovered pulmonary vein conduction as a dominant factor for recurrent atrial tachyarrhythmias after complete circular isolation of the pulmonary veins: lessons from double Lasso technique. Circulation. 2005:111(2):127-35.

2. Verma A, Kilicaslan F, Pisano E, Marrouche NF, Fanelli R, Brachmann J, et al. Response of atrial fibrillation to pulmonary vein antrum isolation is directly related to resumption and delay of pulmonary vein conduction. Circulation. 2005;112(5):627-35.

3. Aryana A, Singh SM, Mugnai G, de Asmundis C, Kowalski M, Pujara DK, et al. Pulmonary vein reconnection following catheter ablation of atrial fibrillation using the second-generation cryoballoon versus open-irrigated radiofrequency: results of a multicenter analysis. J Intervent Cardiac Electrophysiol : Int J Arrhythmias Pacing. 2016:47(3):341-8.

4. Balt JC, Karadavut S, Mulder AA, Luermans JG, Wijffels MC, Boersma LV. Conduction recovery in patients with recurrent atrial fibrillation after pulmonary vein isolation using multi-electrode duty cycled radiofrequency ablation. J Intervent Cardiac Electrophysiol : Int J Arrhythmias Pacing. 2013; 37(2):197-204
5. Callans DJ, Gerstenfeld EP, Dixit S, Zado E, Vanderhoff M, Ren JF, et al. Efficacy of repeat pulmonary vein isolation procedures in patients with recurrent atrial fibrillation. J Cardiovasc Electrophysiol. 2004;15(9):1050-5.

6. Spitzer SG, Leitz P, Langbein A, Karolyi L, Scharfe F, Weinmann T, et al. Circumferential pulmonary vein isolation with second-generation multipolar catheter in patients with paroxysmal or persistent atrial fibrillation: Procedural and one-year follow-up results. Int J Cardiol. 2017;241:212-7.

7. Knight BP, Novak PG, Sangrigoli R, Champagne J, Dubuc M, Adler SW, et al. Long-Term Outcomes After Ablation for Paroxysmal Atrial Fibrillation Using the Second-Generation Cryoballoon: Final Results From STOP AF PostApproval Study. JACC Clin Electrophysiol. 2019;5(3):306-14.

8. Wieczorek M, Hoeltgen R, Brueck M. Does the number of simultaneously activated electrodes during phased RF multielectrode ablation of atrial fibrillation influence the incidence of silent cerebral microembolism? Heart Rhythm. 2013;10(7):953-9.

9. De Greef Y, Dekker L, Boersma L, Murray S, Wieczorek M, Spitzer SG, et al. Low rate of asymptomatic cerebral embolism and improved procedural efficiency with the novel pulmonary vein ablation catheter GOLD: results of the PRECISION GOLD trial. Europace : Eur Pacing, Arrhythmias, Cardiac Electrophysiol : J Working Groups Cardiac Pacing, Arrhythmias, Cardiac Cell Electrophysiol Eur Soc Cardiol. 2016;18(5):687-95.

10. Wieczorek M, Tajtaraghi S, Sassani K, Hoeltgen R. Incidence of early pulmonary vein reconnections using different energy sources for pulmonary vein isolation: Multielectrode phased radiofrequency vs second-generation cryoballoon. J Cardiovasc Electrophysiol. 2019;30(9):1428-35.

11. Gaita F, Leclercq JF, Schumacher B, Scaglione M, Toso E, Halimi F, et al. Incidence of silent cerebral thromboembolic lesions after atrial fibrillation ablation may change according to technology used: comparison of irrigated radiofrequency, multipolar nonirrigated catheter and cryoballoon. J Cardiovasc Electrophysiol. 2011;22(9):961-8.

12. Shah $\mathrm{S}, \mathrm{Xu}$ W, Adelstein E, Voigt A, Saba S, Jain S. Characterization of pulmonary vein reconnection post Cryoballoon ablation. Indian Pacing Electrophysiol J. 2019;19(4):129-33.

13. Rademakers LM, Romero I, Simmers TA, van der Voort PH, Meijer AM, Dekker LR. Spatial distribution of electrical reconnection after pulmonary vein isolation in patients with recurrent paroxysmal atrial fibrillation. Neth Heart J : Mon J Neth Soc Cardiol Neth Heart Found. 2016:24(7-8):481-7.

14. Kistler PM, Ho SY, Rajappan K, Morper M, Harris S, Abrams D, et al. Electrophysiologic and anatomic characterization of sites resistant to electrical isolation during circumferential pulmonary vein ablation for atrial fibrillation: a prospective study. J Cardiovasc Electrophysiol. 2007;18(12): 1282-8.

15. Rajappan K, Kistler PM, Earley MJ, Thomas G, Izquierdo M, Sporton SC, et al. Acute and chronic pulmonary vein reconnection after atrial fibrillation ablation: a prospective characterization of anatomical sites. Pacing Clin Electrophysiol : PACE. 2008;31(12):1598-605.

16. Glowniak A, Tarkowski A, Fic P, Wojewoda K, Wojcik J, Wysokinski A. Second-generation cryoballoon ablation for recurrent atrial fibrillation after an index procedure with radiofrequency versus cryo: Different pulmonary vein reconnection patterns but similar long-term outcomeResults of a multicenter analysis. J Cardiovasc Electrophysiol. 2019;30(7): 1005-12.

17. Chun KR, Schmidt B, Metzner A, Tilz R, Zerm T, Koster I, et al. The 'single big cryoballoon' technique for acute pulmonary vein isolation in patients with paroxysmal atrial fibrillation: a prospective observational single centre study. Eur Heart J. 2009;30(6):699-709.

18. Heeger $\mathrm{CH}$, Wissner E, Mathew S, Deiss S, Lemes C, Rillig A, et al. Once Isolated, Always Isolated? Incidence and Characteristics of Pulmonary Vein Reconduction After Second-Generation Cryoballoon-Based Pulmonary Vein Isolation. Circ Arrhythm Electrophysiol. 2015;8(5):1088-94.

19. Westra SW, van Vugt SPG, Sezer S, Evertz R, Hemels ME, Beukema RJ, et al. Second-generation cryoballoon ablation for recurrent atrial fibrillation after an index cryoballoon procedure: a staged strategy with variable balloon size. J Interv Cardiac Electrophysiol : Int J Arrhythmias Pacing. 2019;54(1):17-24.

20. Chen S, Schmidt B, Bordignon S, Bologna F, Nagase T, Perrotta L, et al. Practical Techniques in Cryoballoon Ablation: How to Isolate Inferior Pulmonary Veins. Arrhythm Electrophysiol Rev. 2018;7(1):11-7.

21. Buist TJ, Adiyaman A, Smit JJJ, Ramdat Misier AR, Elvan A. Arrhythmia-free survival and pulmonary vein reconnection patterns after second-generation 
cryoballoon and contact-force radiofrequency pulmonary vein isolation. Clin Res Cardiol : Official J Ger Cardiac Soc. 2018;107(6):498-506.

22. Nery PB, Belliveau D, Nair GM, Bernick J, Redpath CJ, Szczotka A, et al. Relationship Between Pulmonary Vein Reconnection and Atrial Fibrillation Recurrence: A Systematic Review and Meta-Analysis. JACC Clin Electrophysiol. 2016;2(4):474-83.

23. Miyazaki S, Taniguchi H, Hachiya H, Nakamura H, Takagi T, Hirao K, et al. Clinical recurrence and electrical pulmonary vein reconnections after second-generation cryoballoon ablation. Heart Rhythm. 2016;13(9):1852-7.

24. Dixit S, Gerstenfeld EP, Ratcliffe SJ, Cooper JM, Russo AM, Kimmel SE, et al. Single procedure efficacy of isolating all versus arrhythmogenic pulmonary veins on long-term control of atrial fibrillation: a prospective randomized study. Heart Rhythm. 2008;5(2):174-81.

25. Duytschaever M, Anne W, Papiashvili G, Vandekerckhove Y, Tavernier R. Mapping and isolation of the pulmonary veins using the PVAC catheter. Pacing Clin Electrophysiol : PACE. 2010;33(2):168-78.

26. Conte G, Soejima K, de Asmundis C, Chierchia GB, Badini M, Miwa Y, et al. Value of high-resolution mapping in optimizing cryoballoon ablation of atrial fibrillation. Int J Cardiol. 2018;270:136-42

\section{Publisher's Note}

Springer Nature remains neutral with regard to jurisdictional claims in published maps and institutional affiliations.

Ready to submit your research? Choose BMC and benefit from:

- fast, convenient online submission

- thorough peer review by experienced researchers in your field

- rapid publication on acceptance

- support for research data, including large and complex data types

- gold Open Access which fosters wider collaboration and increased citations

- maximum visibility for your research: over $100 \mathrm{M}$ website views per year

At BMC, research is always in progress.

Learn more biomedcentral.com/submissions 